NEETs (HR: 2.5 95\% CI 2.2 to 2.9). The elevated risk remained even when the models were fitted separately by gender.

Conclusion We found that NEET experiences are associated with elevated risks of mortality, hospitalisation and poor mental health. Disengagement from employment and education during transition from school to work may lead to long term negative health effects which in turn results in social and economic costs to society. Policy intervention is necessary in assisting NEET young people to re-engage in education or employment.

\section{P11 MATHEMATICAL COUPLING AND CAUSAL INFERENCE THROUGH EXAMPLE}

${ }^{1}$ L Berrie*, 'PWG Tennant, ${ }^{2}$ PD Norman, ${ }^{1}$ PD Baxter, ${ }^{1}$ MS Gilthorpe. ${ }^{1}$ School of Medicine, University of Leeds, Leeds, UK; ${ }^{2}$ School of Geography, University of Leeds, Leeds, UK

\subsection{6/jech-2018-SSMabstracts. 137}

Background In health studies, proportions and percentages can often seem more informative than raw counts and therefore appear to be of more interest to analysts. However, it has long been acknowledged that their use is problematic in correlation and regression analyses where they comprise common components that are present in both the dependent and independent constituents of a model (exposure and outcome), as in the regression analysis of proportions with common denominators. We demonstrate this so-called mathematical coupling with real-world examples aided by directed acyclic graphs (DAGs) and simulations.

Methods We consider three possible real-world scenarios: (1) the population size $(\mathrm{N})$ of a geographical area causes both the number of people living in detached houses (X) and the number of people living in care homes $(\mathrm{Y})$, within each area, but the number of detached houses (X) does not cause the number of care homes (Y) within any area, or vice versa; (2) the population size $(\mathrm{N})$ of a geographical area causes both the number of people with no formal qualifications $(\mathrm{X})$ and the number of people with poor self-reported health $(\mathrm{Y})$, while both the population size $(\mathrm{N})$ and number of people with no formal qualifications (X) are causes of the number of people with self-reported poor health (Y); and (3) within a geographical area, the area wealth $(\mathrm{X})$ causes the number of elderly people $(\mathrm{N})$, while both area wealth $(\mathrm{X})$ and the number of elderly people $(\mathrm{N})$ cause social care expenditure $(\mathrm{Y})$.

Results We show how historical solutions to the issue of mathematical coupling caused by a common denominator hold under the situation when the denominator is a confounder of the exposure outcome relationship; i.e. the results of the simulated examples under scenarios 1 and 2 result in expected regression coefficients. The same solution does not hold in scenario 3, when the denominator is a mediator (i.e. lies on the causal path) between the exposure and outcome.

Conclusion We show how DAGs and accompanying causal graph theory can be used to understand a problem first presented over a century ago. We highlight the issue of mathematical coupling when analysing proportions with a common denominator, showing under which circumstances historical solutions are valid or invalid. By using real-world examples to inform simulations, we demonstrate the utility of DAGs and causal graph theory in health geography and observational research to understand statistical problems and to verify proposed solutions.

\section{P12 HOW TO BUILD THE 'RIGHT' DIRECTED ACYCLIC GRAPH (DAG): A SYSTEMATIC, TRANSPARENT AND ACCESSIBLE METHOD FOR EVIDENCE SYNTHESIS}

${ }^{1} \mathrm{KD}$ Ferguson*, ${ }^{2} \mathrm{~J}$ Lewsey, ${ }^{1} \mathrm{M}$ McCann, ${ }^{3} \mathrm{D}$ Smith. ${ }^{1} \mathrm{M} R \mathrm{C} / \mathrm{CSO}$ Social and Public Health Sciences Unit, University of Glasgow, Glasgow, UK; ${ }^{2}$ Health Economics and Health Technology Assessment, University of Glasgow, Glasgow, UK; ${ }^{3}$ Mental Health and Wellbeing, University of Glasgow, Glasgow, UK

\subsection{6/jech-2018-SSMabstracts. 138}

Background Causal inference methods are increasingly popular in health research, with directed acyclic graphs (DAGs) being notably prominent. Theoretically, DAGs are powerful tools for minimising bias in quantitative analysis, however their transition into practice has been problematic. Lack of guidelines for generating the 'right' DAG for research questions have been cited as a central reason. This study presents a solution in the form of 'evidence synthesis for constructing directed acyclic graphs' (ESC-DAGs). The approach embeds DAGs in a procedural evidence synthesis method which focuses on how to derive and integrate DAGs from research evidence in a transparent and systematic fashion.

Methods For studies meeting inclusion criteria: 1) Appraisal of study quality with split focus on the degree of explicit causal thinking employed and on more generic study quality issues such as study design, sample size, etc; 2) Mapping of conclusions for each study using causal inference theory to produce an 'implied graph'; 3) Translation of implied graphs into DAGs through procedural application of four 'causal criteria' to each relationship in the implied graph (temporality, plausibility, recourse to theory, counterfactual thought experiment); 4) Integration of DAGs, starting with those with the highest appraisal scores until all DAGs are integrated. The output is an 'integrated-DAG'. ESC-DAGs is demonstrated on the exposure-outcome relationship of parental influences on adolescent alcohol harm.

Results 30 studies were included. Study appraisal produces a scale with scores ranging from 0 to 5 (median=2). The DAGs produced for individual studies are substantially less comprehensive than the integrated-DAG (covering between 5\% and $40 \%$ of causal pathways). Over $90 \%$ of the implied graphs were changed during the translation process. The most common changes reflect a strong tendency in research to either mistakenly control for mediation or for unjustified control of parallel risk factors.

Conclusion As a methodological contribution to an increasingly popular form of health research, ESC-DAGs has broad relevance to population health. Through its systematic treatment of research evidence, ESC-DAGs is a reproducible and transparent process that is suitable for use by researchers with only minimal training on the causal inference methods. Compared to how DAGs have been constructed elsewhere, those generated from ESC-DAGs are more comprehensive and have greater potential to reduce bias. In meeting the need for guidelines on generating DAGs in such a way, ESC-DAGs represents an important step towards realising the potential of DAGs to improve the practice of health research. 